\title{
ON CERTAIN SLOWLY CONVERGENT SERIES OCCURRING IN PLATE CONTACT PROBLEMS
}

\author{
WALTER GAUTSCHI
}

ABSTRACT. A simple computational procedure is developed for accurately summing series of the form $\sum_{k=0}^{\infty}(2 k+1)^{-p} z^{2 k+1}$, where $z$ is complex with $|z| \leq 1$ and $p=2$ or 3 , as well as series of the type

$$
\sum_{k=0}^{\infty}(2 k+1)^{-p} \cosh (2 k+1) x / \cosh (2 k+1) b
$$

and

$$
\sum_{k=0}^{\infty}(2 k+1)^{-p} \sinh (2 k+1) x / \cosh (2 k+1) b
$$

where $0 \leq x \leq b, p=2$ or 3 . The procedures are particularly useful in cases where the series converge slowly. Numerical experiments illustrate the effectiveness of the procedures.

\section{INTRODUCTION}

Our concern, in $\S \S 2-4$, is with series of the type

$$
R_{p}(z)=\sum_{k=0}^{\infty} \frac{z^{2 k+1}}{(2 k+1)^{p}}
$$

or the type

$$
S_{p}(z)=\sum_{k=0}^{\infty}(-1)^{k} \frac{z^{2 k+1}}{(2 k+1)^{p}}
$$

where

$$
z \in \mathbb{C}, \quad|z| \leq 1, \quad \text { and } p=2 \text { or } 3 .
$$

Of particular interest to us is the numerical evaluation of these series in cases of slow convergence, i.e., when $|z|$ is close or equal to 1 . It clearly suffices to concentrate on the first of the two series, $R_{p}$, since

$$
S_{p}(z)=i R_{p}(-i z) .
$$

Received July 23, 1990.

1980 Mathematics Subject Classification (1985 Revision). Primary 40A25; Secondary 44A10, $33 \mathrm{~A} 65$.

Key words and phrases. Slowly convergent series, Laplace transformation, Stieltjes transform, orthogonal polynomials.

Work supported, in part, by the National Science Foundation under grant CCR-8704404. 
Furthermore, $R_{p}(-z)=-R_{p}(z)$ and $R_{p}(\bar{z})=\overline{R_{p}(z)}$, so that attention can be restricted to the first quadrant of the complex plane.

Series of the type $\left(1.1_{p}\right)$, with

$$
z=A, \quad 0<A \leq 1, \quad \text { and } \quad z=e^{i \alpha}, \quad \alpha \in \mathbb{R},
$$

occur in the mathematical treatment of unilateral plate contact problems, and their numerical evaluation, in this context, has recently been discussed by $\mathrm{K}$. M. Dempsey, D. Liu, and J. P. Dempsey [1]. The method proposed by these authors consists in applying Plana's summation formula, which in turn requires the numerical evaluation of several definite integrals-for example by Romberg integration.

Here we develop a technique which appears to be considerably simpler. All it requires is the application (in the backward direction) of a three-term recurrence relation, once a set of numerical constants has been precomputed. Results of high accuracy are easily achieved, even for $|z|$ near or equal to 1 .

Some of the series $\left(1.1_{p}\right),\left(1.2_{p}\right)$ with $p=2$ or $p=3$ can be summed explicitly as Fourier series when $z$ is given by the second expression in (1.5). We thus have

$$
\begin{aligned}
& \text { (1.6 }) \sum_{k=0}^{\infty} \frac{\cos (2 k+1) \alpha}{(2 k+1)^{2}}=\pi(\pi-2|\alpha|) / 8, \quad-\pi \leq \alpha \leq \pi \quad[8,(17.2 .16)], \\
& \text { (1.6. }) \sum_{k=0}^{\infty} \frac{\sin (2 k+1) \alpha}{(2 k+1)^{3}}=\pi \alpha(\pi-|\alpha|) / 8, \quad-\pi \leq \alpha \leq \pi \quad[8,(14.2 .21)],
\end{aligned}
$$

and analogous formulae for

$$
\sum_{k=0}^{\infty}(-1)^{k}(2 k+1)^{-2} \sin (2 k+1) \alpha, \quad \sum_{k=0}^{\infty}(-1)^{k}(2 k+1)^{-3} \cos (2 k+1) \alpha,
$$

which can be obtained from (1.6) by applying (1.4). When $z=1$, the sum of $\left(1.1_{p}\right)$ is expressible in terms of the Riemann zeta function,

$$
R_{p}(1)=\left(1-2^{-p}\right) \zeta(p),
$$

whereas $S_{2}(1)$ is known as Catalan's constant, and $S_{3}(1)=\pi^{3} / 32$. All these explicit formulae will be useful for testing purposes.

In $\S 5$ we combine our techniques of $\S 2$ with series expansion to deal with the more difficult series

$$
T_{p}(x, b)=\sum_{k=0}^{\infty} \frac{1}{(2 k+1)^{p}} \frac{\cosh (2 k+1) x}{\cosh (2 k+1) b}
$$

and

$$
U_{p}(x, b)=\sum_{k=0}^{\infty} \frac{1}{(2 k+1)^{p}} \frac{\sinh (2 k+1) x}{\cosh (2 k+1) b}
$$


where

$$
0 \leq x \leq b, \quad b>0, \quad \text { and } \quad p=2,3 .
$$

Both are also of interest in plate contact problems [1]. Here again, we are able to sum these series effectively and to high precision, the major (as yet unresolved) difficulty occurring when $b$ is very small.

$$
\text { 2. Summation of } R_{p} \text { AND } S_{p}, p=2 \text { AND } p=3
$$

We begin with an idea used previously in [7,6], namely to express part of each term of the series (not the whole term, as in [7, 6]) as a Laplace transform with integer argument. Specifically,

$$
\frac{1}{(k+1 / 2)^{p}}=(\mathscr{L} f)(k), \quad \mathscr{L}=\text { Laplace transform }
$$

where

$$
f(t)=\frac{1}{(p-1) !} t^{p-1} e^{-t / 2}
$$

Then

$$
\begin{aligned}
R_{p}(z) & =\frac{z}{2^{p}} \sum_{k=0}^{\infty} \frac{z^{2 k}}{(k+1 / 2)^{p}}=\frac{z}{2^{p}} \sum_{k=0}^{\infty} z^{2 k} \int_{0}^{\infty} e^{-k t} \cdot \frac{t^{p-1} e^{-t / 2}}{(p-1) !} d t \\
& =\frac{z}{2^{p}(p-1) !} \int_{0}^{\infty} \sum_{k=0}^{\infty}\left(z^{2} e^{-t}\right)^{k} \cdot t^{p-1} e^{-t / 2} d t \\
& =\frac{z}{2^{p}(p-1) !} \int_{0}^{\infty} \frac{1}{1-z^{2} e^{-t}} t^{p-1} e^{-t / 2} d t
\end{aligned}
$$

that is,

$$
R_{p}(z)=\frac{z}{2^{p}(p-1) !} \int_{0}^{\infty} \frac{t^{p-1} e^{t / 2}}{e^{t}-z^{2}} d t
$$

We distinguish two cases.

Case 1: $z=1$. In this case, (2.3) takes the form

$$
R_{p}(1)=\frac{1}{2^{p}(p-1) !} \int_{0}^{\infty} \frac{t}{e^{t}-1} \cdot t^{p-2} e^{t / 2} d t
$$

and can be evaluated by Gaussian quadrature relative to the weight function (cf. [7])

$$
\varepsilon(t)=\frac{t}{e^{t}-1} \text { on }[0, \infty] \quad \text { ("Einstein function"). }
$$

However, there is no real need for this, since by (1.7) the sum is expressible in terms of the well-tabulated Riemann zeta function [9]. In particular, $R_{2}(1)=$ $\pi^{2} / 8$. 
The more difficult case is

Case 2: $z \neq 1$. Here we could proceed similarly as in (2.4) and write

$$
R_{p}(z)=\frac{z}{2^{p}(p-1) !} \int_{0}^{\infty} \varepsilon(t) \cdot \frac{e^{t}-1}{e^{t}-z^{2}} \cdot t^{p-2} e^{t / 2} d t .
$$

Unfortunately, the second factor in the integrand is quite ill-behaved when $|z|$ is close to 1, exhibiting a steep boundary layer near $t=0$. (Consider, e.g., $z^{2}=1-\eta, 0<\eta \ll 1$.) Gaussian quadrature, therefore, will no longer be effective.

Instead, we make the change of variable $e^{-t}=\tau$ in (2.3) (and then replace $\tau$ again by $t$ ) to obtain

$$
R_{p}(z)=\frac{1}{2^{p}(p-1) ! z} \int_{0}^{1} \frac{[\ln (1 / t)]^{p-1}}{\sqrt{t}} \frac{d t}{z^{-2}-t} .
$$

This expresses $R_{p}(z)$ as a Stieltjes transform of the weight function

$$
w_{p}(t)=\frac{[\ln (1 / t)]^{p-1}}{\sqrt{t}} \text { on }[0,1] .
$$

Our assumptions on $z$ are such that the point $z^{-2}$ at which the transform is evaluated lies outside of the interval $[0,1]$,

$$
z^{-2} \in \mathbb{C} \backslash[0,1] \text {. }
$$

The integral in (2.7), therefore, can be evaluated by backward recursion, as is discussed in $[3, \S 5]$.

Indeed, if

$$
y_{n+1}=\left(z^{-2}-\alpha_{n}\right) y_{n}-\beta_{n} y_{n-1}, \quad n=0,1,2, \ldots,
$$

is the recurrence relation for the orthogonal polynomials $\left\{\pi_{n}\left(z^{-2} ; w_{p}\right)\right\}$ relative to the weight function (2.8), thus,

$$
\alpha_{n}=\alpha_{n}\left(w_{p}\right), \quad \beta_{n}=\beta_{n}\left(w_{p}\right) \quad\left[\beta_{0}\left(w_{p}\right)=\int_{0}^{1} w_{p}(t) d t\right],
$$

and if we define the sequence $\left\{r_{n-1}^{[\nu]}(z)\right\}_{n=0}^{\nu+1}$ for any integer $\nu>0$ by

$$
r_{\nu}^{[\nu]}(z)=0, \quad r_{n-1}^{[\nu]}(z)=\frac{\beta_{n}}{z^{-2}-\alpha_{n}-r_{n}^{[\nu]}(z)}, \quad n=\nu, \nu-1, \ldots, 1,0,
$$

then (cf. [3, equation (5.2) for $N=0$ ])

$$
\int_{0}^{1} \frac{w_{p}(t) d t}{z^{-2}-t}=\lim _{\nu \rightarrow \infty} r_{-1}^{[\nu]}(z)
$$

Thus, by (2.7),

$$
R_{p}(z)=\frac{r_{-1}^{[\infty]}(z)}{2^{p}(p-1) ! z}
$$


Convergence in (2.13) is faster the further away $z^{-2}$ is from the interval $[0,1]$.

The evaluation of $r_{-1}^{[\infty]}(z)$ is quite cheap, once the coefficients $\alpha_{n}, \beta_{n}$ in (2.11) have been precomputed for sufficiently many $n$. One simply lets $\nu$ increase through a sequence $\left\{\nu_{i}\right\}$ of integers $0<\nu_{1}<\nu_{2}<\cdots$ and stops at the smallest $i$, say $i=i_{\text {min }}$, for which $\left|r_{-1}^{\left[\nu_{i}\right]}(z)-r_{-1}^{\left[\nu_{i-1}\right]}(z)\right| \leq \varepsilon\left|r_{-1}^{\left[\nu_{i}\right]}(z)\right|$, where $\varepsilon$ is a preset error tolerance. One then accepts $r_{-1}^{\left[\nu_{i}\right]}(z)$ with $i=i_{\min }$ as the desired approximation of $r_{-1}^{[\infty]}(z)$ in (2.14). For the two choices of $z$ in (1.5), practical guidelines for determining an acceptable value of $\nu$ (i.e., one for which $r_{-1}^{[\nu]}(z)$ sufficiently approximates $\left.r_{-1}^{[\infty]}(z)\right)$ will be given in $\S 4$.

The coefficients $\alpha_{n}, \beta_{n}$ can be computed by known methods, as will be further discussed in $\S 3$. The first 100 coefficients are tabulated in Tables 1 and 2 of the Appendix for $p=2$ and $p=3$ to an accuracy of 25 and 20 significant decimal digits, respectively.

The procedure (2.12)-(2.14), in view of (1.4), is readily adapted to the series $S_{p}$ in $\left(1.2_{p}\right)$. Indeed, letting $s_{n}^{[\nu]}(z)=-r_{n}^{[\nu]}(-i z)$, one finds

$$
S_{p}(z)=\frac{s_{-1}^{[\infty]}(z)}{2^{p}(p-1) ! z}
$$

where

$$
s_{\nu}^{[\nu]}(z)=0, \quad s_{n-1}^{[\nu]}(z)=\frac{\beta_{n}}{z^{-2}+\alpha_{n}-s_{n}^{[\nu]}(z)}, \quad n=\nu, \nu-1, \ldots, 1,0 .
$$

Since $S_{p}(z)$ is effectively the Stieltjes transform of $w_{p}(\cdot)$ evaluated at $-z^{-2}$, the process $(2.15),(2.16)$ converges more rapidly (as $\nu \rightarrow \infty)$ the further away $-z^{-2}$ is from the interval $[0,1]$. In particular, it converges rapidly for $z=1$, yielding a fast way of computing Catalan's constant when $p=2$. Indeed, taking $\nu=16$ in $(2.16)$ produces $s_{-1}^{[\infty]}(1)$, hence $S_{2}(1)$, accurately to 25 decimal digits!

3. Generating the COefficients $\alpha_{n}\left(w_{p}\right), \beta_{n}\left(w_{p}\right)$ FOR $p=2$ AND $p=3$

Consider the weight function

$$
w_{p}(t ; \alpha)=t^{\alpha}[\ln (1 / t)]^{p-1}, \quad 0<t \leq 1, \alpha>-1, p \geq 2,
$$

and let

$$
m_{n}(\alpha ; p)=\int_{0}^{1} P_{n}^{*}(t) w_{p}(t ; \alpha) d t, \quad n=0,1,2, \ldots,
$$

denote the "modified moments" of $w_{p}(\cdot ; \alpha)$ relative to the shifted Legendre polynomials $P_{n}^{*}(t)=P_{n}(2 t-1)$. In the case $p=2$ these modified moments 
are explicitly known (cf. [2]):

$$
\begin{aligned}
m_{n}(\alpha ; 2)= & \frac{1}{\alpha+1}\left\{\frac{1}{\alpha+1}+2 \sum_{k=1}^{n} \frac{k}{(k+\alpha+1)(k-\alpha-1)}\right\} \\
& \cdot \prod_{k=1}^{n} \frac{\alpha+1-k}{\alpha+1+k} .
\end{aligned}
$$

It is also well known how the modified moments of a weight function $w$ can be used to generate the recursion coefficients $\alpha_{n}(w), \beta_{n}(w)$ of the respective orthogonal polynomials $\left\{\pi_{k}(\cdot ; w)\right\}$ by means of the so-called modified Chebyshev algorithm $[4, \S 2.4]$. This algorithm indeed works particularly well in the case of the weight function (3.1) with $p=2, \alpha=-\frac{1}{2}$, i.e., for $w(t)=w_{2}(t)$ (cf. (2.8)), as was demonstrated in [5, Example 5.3]. This, then, is the way we computed the quantities $\alpha_{n}\left(w_{p}\right), \beta_{n}\left(w_{p}\right)$ for $p=2$. Compensating for a loss of about four decimal digits, when $n$ runs from 0 to 99 , we tabulate the results in Table 1 of the Appendix to only 25 decimals (having done the computation in 29-decimal arithmetic).

In order to get the same quantities for $p=3$, it suffices to observe that

$$
\frac{\partial w_{p}}{\partial \alpha}(t ; \alpha)=-w_{p+1}(t ; \alpha)
$$

and therefore

$$
m_{n}(\alpha ; p+1)=-\int_{0}^{1} P_{n}^{*}(t) \frac{\partial w_{p}}{\partial \alpha}(t ; \alpha) d t=-\frac{\partial m_{n}}{\partial \alpha}(\alpha ; p) .
$$

Thus, the required modified moments $m_{n}(\alpha ; 3)$ can be obtained by differentiating both sides of (3.3) with respect to $\alpha$ (after multiplication by -1 ). The result is

$$
\begin{aligned}
m_{n}(\alpha ; 3)=\frac{2}{(\alpha+1)^{3}}\{1+ & 2(\alpha+1) \sum_{1} \\
& \left.+2(\alpha+1)^{2} \sum_{1}^{2}-2(\alpha+1)^{3} \sum_{2}\right\} \Pi,
\end{aligned}
$$

where

$$
\begin{aligned}
\sum_{1} & =\sum_{k=1}^{n} \frac{k}{(k+\alpha+1)(k-\alpha-1)}, \\
\sum_{2} & =\sum_{k=1}^{n} \frac{k}{(k+\alpha+1)^{2}(k-\alpha-1)^{2}}, \\
\Pi & =\prod_{k=1}^{n} \frac{\alpha+1-k}{\alpha+1+k} .
\end{aligned}
$$

Putting $\alpha=-\frac{1}{2}$ in (3.4), and using the resulting quantities as input to the modified Chebyshev algorithm, produces the coefficients $\alpha_{n}\left(w_{3}\right), \beta_{n}\left(w_{3}\right)$. The procedure is somewhat less stable than in the case $p=2$, suffering a loss of 
about eight to nine decimal digits when applied up to $n=99$. For this reason we tabulate $\alpha_{n}\left(w_{3}\right), \beta_{n}\left(w_{3}\right)$ in Table 2 of the Appendix to only 20 decimals.

\section{IMPLEMENTATION AND NUMERICAL EXAMPLES}

It would clearly be desirable in our procedure (2.12) to know a priori what value to choose for the starting index $\nu$, given any $z$ in the first quadrant of $\mathbb{C}$ and given the required accuracy. The recursion in (2.12) then would need to be run through only once, and the iterative procedure suggested in $\S 2$ could be dispensed with.

To deal with this problem, we consider only the two cases of practical interest stated in (1.5). More precisely, we address the following related problem: Given $\nu$ and the desired relative accuracy $\varepsilon$, determine the set of values $A$ in $[0,1]$, resp. $\alpha$ in $[0, \pi / 2]$, for which $r_{-1}^{[\nu]}$ in $(2.13)$ approximates $r_{-1}^{[\infty]}$ within a relative error of $\varepsilon$.

As to the values of $A$, we note that the speed of convergence in (2.13) decreases as $A$ increases in $[0,1]$. The desired set of $A$-values must thus have the form $0 \leq A \leq A(\nu, \varepsilon) \leq 1$, and the problem is to determine $A(\nu, \varepsilon)$. We solve this empirically by a bisection procedure: Start with two numbers $A_{0}^{-}, A_{0}^{+}$such that $A_{0}^{-} \leq A(\nu, \varepsilon) \leq A_{0}^{+}$, for example, $A_{0}^{-}=0, A_{0}^{+}=1$. Having already obtained $A_{k-1}^{-}$and $A_{k-1}^{+}$with $A_{k-1}^{-}<A_{k-1}^{+}$, test the midpoint $M=\frac{1}{2}\left(A_{k-1}^{-}+A_{k-1}^{+}\right)$to see whether at $M$ the procedure (2.12) yields an approximation $r_{-1}^{[\nu]}$ with relative error larger or smaller than $\varepsilon$. In the former case we set $A_{k}^{-}=A_{k-1}^{-}, A_{k}^{+}=M$, in the latter case $A_{k}^{-}=M, A_{k}^{+}=A_{k-1}^{+}$. We quit this iteration as soon as, say, $A_{k}^{+}-A_{k}^{-} \leq \frac{1}{2} 10^{-6}$ and take $\frac{1}{2}\left(A_{k}^{-}+A_{k}^{+}\right)$ to approximate $A(\nu, \varepsilon)$. In order to determine the relative errors of $r_{-1}^{[\nu]}$, as required in this procedure, we approximate $r_{-1}^{[\infty]}$ by $r_{-1}^{[99]}$ and, at the same time, check to see that $r_{-1}^{[99]}$ and $r_{-1}^{[98]}$ agree to within a relative accuracy $\varepsilon / 100$. If they do, it is safe to assume that $r_{-1}^{[99]}$ can reliably substitute $r_{-1}^{[\infty]}$ in determining whether $r_{-1}^{[\nu]}$ has relative error $>\varepsilon$ or $<\varepsilon$. If they do not, we print a cautionary message, and take $A_{k}^{-}$as a (conservative) estimate from below of $A(\nu, \varepsilon)$.

The results of this procedure are summarized in Table 4.1 for both $p=2$ and $p=3$. An asterisk indicates a conservative lower estimate of $A(\nu, \varepsilon)$ for reasons explained above.

We can see from Table 4.1, for example, that if we are interested in 12-digit accuracy and only in positive values of $A$ satisfying $A \leq .99$, then we can safely use $\nu=50$ in (2.12) when $p=2$, and $\nu=40$ when $p=3$. On the other hand, the choice $\nu=10$ for the same range of $A$-values, always gives at least four correct decimal digits.

Interestingly, the procedure (2.12), (2.13) seems to converge even for $A=1$, albeit slowly, but there is no theoretical justification for it (to our knowledge). 
TABLE 4.1

Values of $A(\nu, \varepsilon), \varepsilon=\frac{1}{2} 10^{-a c c}$, such that $r_{-1}^{[\nu]}$ approximates $r_{-1}^{[\infty]}$ to acc digits for all $A$ with $0 \leq A \leq A(\nu, \varepsilon)$

\begin{tabular}{|rrrrrrrrrrrr|}
\hline$\nu$ & $a c c$ & $p=2$ & $p=3$ & $\nu$ & $a c c$ & $p=2$ & $p=3$ & $\nu$ & $a c c$ & $p=2$ & $p=3$ \\
\hline 10 & 4 & .9902 & 1.0000 & 40 & 4 & .9999 & 1.0000 & 70 & 4 & 1.0000 & 1.0000 \\
& 8 & .9313 & .9592 & & 8 & .9962 & .9993 & & 8 & .9989 & 1.0000 \\
& 12 & .8422 & .8732 & & 12 & .9889 & .9936 & & 12 & $.9961^{*}$ & $.9980^{*}$ \\
& 16 & .7384 & .7688 & & 16 & .9786 & .9842 & & 16 & $.9922^{*}$ & .9955 \\
& 20 & .6325 & .6603 & & 20 & .9653 & .9717 & & 20 & .9887 & .9914 \\
20 & 4 & .9985 & 1.0000 & 50 & 4 & 1.0000 & 1.0000 & 80 & 4 & 1.0000 & 1.0000 \\
& 8 & .9827 & .9931 & & 8 & .9977 & .9998 & & 8 & $.9990^{*}$ & 1.0000 \\
& 12 & .9551 & .9685 & & 12 & .9931 & .9963 & & 12 & $.9961^{*}$ & $.9980^{*}$ \\
& 16 & .9178 & .9330 & & 16 & .9864 & .9903 & & 16 & $.9922^{*}$ & $.9961^{*}$ \\
& 20 & .8729 & .8891 & & 20 & .9777 & .9823 & & 20 & .9914 & $.9922^{*}$ \\
30 & 4 & .9996 & 1.0000 & 60 & 4 & 1.0000 & 1.0000 & 90 & 4 & 1.0000 & 1.0000 \\
& 8 & .9927 & .9980 & & 8 & .9985 & 1.0000 & & 8 & $.9990^{*}$ & 1.0000 \\
& 12 & .9800 & .9873 & & 12 & .9953 & .9976 & & 12 & $.9961^{*}$ & $.9980^{*}$ \\
& 16 & .9620 & .9708 & & 16 & .9906 & .9936 & & 16 & $.9922^{*}$ & $.9961^{*}$ \\
& 20 & .9395 & .9492 & & 20 & .9845 & .9880 & & 20 & $.9922^{*}$ & $.9922^{*}$ \\
\hline
\end{tabular}

For the second choice $z=e^{i \alpha}, 0 \leq \alpha \leq \pi / 2$, in (1.5), it was observed empirically that the speed of convergence in (2.13) decreases-slowly at first, and then faster-as $\alpha$ decreases from $\pi / 2$ to 0 . Therefore, a similar procedure as above for $A$-values can be applied to determine the number $\omega(\nu, \varepsilon)$ with the property that for all $\alpha$ satisfying $0 \leq \omega(\nu, \varepsilon) \pi / 2 \leq \alpha \leq \pi / 2$, the procedure (2.12) produces $r_{-1}^{[\nu]}$ with (at least approximately) $\left|\left(r_{-1}^{[\nu]}-r_{-1}^{[\infty]}\right) / r_{-1}^{[\infty]}\right| \leq \varepsilon$. The results are displayed in Table 4.2 .

TABLE 4.2

Values of $\omega(\nu, \varepsilon), \varepsilon=\frac{1}{2} 10^{-a c c}$, such that $r_{-1}^{[\nu]}$ approximates $r_{-1}^{[\infty]}$ to acc digits for all $\alpha$ with $\omega(\nu, \varepsilon) \pi / 2 \leq \alpha \leq \pi / 2$

\begin{tabular}{|rrrrrrrrrrrr|}
\hline \multicolumn{1}{|c}{$a c c$} & $p=2$ & $p=3$ & $\nu$ & $a c c$ & $p=2$ & $p=3$ & $\nu$ & acc & $p=2$ & $p=3$ \\
\hline 10 & 4 & .0159 & 0.0000 & 40 & 4 & .0002 & 0.0000 & 70 & 4 & 0.0000 & 0.0000 \\
& 8 & .1066 & .0690 & & 8 & .0054 & .0013 & & 8 & $.0020^{*}$ & 0.0000 \\
& 12 & .2864 & .2313 & & 12 & .0152 & .0096 & & 12 & .0046 & .0025 \\
& 16 & .6789 & .5576 & & 16 & .0294 & .0226 & & 16 & .0092 & $.0078^{*}$ \\
& 20 & $1.0000^{*}$ & $1.0000^{*}$ & & 20 & .0482 & .0404 & & 20 & $.0156^{*}$ & .0120 \\
20 & 4 & .0026 & 0.0000 & 50 & 4 & .0001 & 0.0000 & 80 & 4 & 0.0000 & 0.0000 \\
& 8 & .0247 & .0118 & & 8 & .0033 & .0005 & & 8 & $.0020^{*}$ & 0.0000 \\
& 12 & .0649 & .0481 & & 12 & .0095 & .0056 & & 12 & $.0039^{*}$ & $.0020^{*}$ \\
& 16 & .1251 & .1045 & & 16 & .0185 & .0138 & & 16 & $.0078^{*}$ & $.0078^{*}$ \\
& 20 & .2095 & .1844 & & 20 & .0304 & .0249 & & 20 & $.0156^{*}$ & $.0089^{*}$ \\
30 & 4 & .0007 & 0.0000 & 60 & 4 & 0.0000 & 0.0000 & 90 & 4 & 0.0000 & 0.0000 \\
& 8 & .0103 & .0036 & & 8 & .0022 & .0002 & & 8 & $.0020^{*}$ & 0.0000 \\
& 12 & .0278 & .0190 & & 12 & .0064 & .0036 & & 12 & $.0039^{*}$ & $.0020^{*}$ \\
& 16 & .0535 & .0428 & & 16 & .0126 & .0091 & & 16 & $.0078^{*}$ & $.0078^{*}$ \\
& 20 & .0878 & .0754 & & 20 & .0208 & .0168 & & 20 & $.0156^{*}$ & $.0078^{*}$ \\
\hline
\end{tabular}


TABLE 4.3

Results for Example 1

\begin{tabular}{|ccclc|}
\hline$A$ & $p=2$ & $p=3$ & \multicolumn{1}{c|}{$R_{2}(A)$} & \multicolumn{1}{c|}{$R_{3}(A)$} \\
\hline .8 & 21 & 16 & .8772880939214647253008518 & .82248858052014232615 \\
.9 & 30 & 23 & 1.025938951111110172771877 & .93414857586540185586 \\
.95 & 43 & 31 & 1.114099577929052481501213 & .99191543992242877550 \\
.99 & 95 & 65 & 1.202075664776857538062901 & 1.0395722318736413458 \\
.999 & - & - & 1.2293981974 & 1.0505677498304 \\
1.000 & - & - & 1.2336 & 1.051799789 \\
\hline
\end{tabular}

Example 1. $R_{p}(A)$ for $A=.8, .9, .95, .99, .999,1.000$, and $p=2,3$.

We applied the procedure (2.12) with $\nu=1,2,3, \ldots$, terminating it for the first value of $\nu, \nu=\nu_{\min }$, for which $\left|\left(r_{-1}^{[\nu]}-r_{-1}^{[\nu-1]}\right) / r_{-1}^{[\nu]}\right| \leq \varepsilon$, where $\varepsilon=$ $\frac{1}{2} 10^{-25}$ for $p=2$, and $\varepsilon=\frac{1}{2} 10^{-20}$ for $p=3$. Table 4.3 shows the values of $\nu_{\min }$ along with 25-, resp. 20-digit results for $R_{p}(A), p=2,3$.

For $A \geq .999$, full accuracy could not be achieved with $\nu \leq 99$, only the partially accurate results shown in Table 4.3.

Example 2. $R_{p}\left(e^{i \alpha}\right)$ for $\alpha=\omega \pi / 2, \omega=.2, .1, .05, .01, .001,0.000$, and $p=2,3$.

The same experiment as in Example 1 was run in this case, with the results being shown in Table 4.4. The first entry under each heading $R_{p}\left(e^{i \omega \pi / 2}\right)$ represents the real part, the second the imaginary part. The results for $\operatorname{Re} R_{2}$, $\operatorname{Im} R_{3}$ were checked against formulas $\left(1.6_{2}\right)$ and $\left(1.6_{3}\right)$, respectively, and revealed agreement to all digits shown.

TABLE 4.4

Results for Example 2

\begin{tabular}{|c|c|c|c|c|}
\hline$\omega$ & $p=2$ & $p=3$ & $R_{2}\left(e^{i \omega \pi / 2)}\right)$ & $R_{3}\left(e^{i \omega \pi / 2}\right)$ \\
\hline .2 & 27 & 21 & $\begin{array}{l}.9869604401089358618834491 \\
.4474022700859631972532577\end{array}$ & $\begin{array}{l}.96915102126251836837 \\
.34882061265337297697\end{array}$ \\
\hline .1 & 37 & 28 & $\begin{array}{c}1.110330495122552844618880 \\
.2783029792855803918158969\end{array}$ & $\begin{array}{r}1.0268555576593748316 \\
.18409976778928018229\end{array}$ \\
\hline .05 & 51 & 38 & $\begin{array}{c}1.172015522629361335986596 \\
.1663915239689736941195221\end{array}$ & $\begin{array}{r}1.0444944153967221625 \\
.09447224926028851460\end{array}$ \\
\hline .01 & - & 76 & $\begin{array}{c}1.2213635446348081290808 \\
.04592009281744058404956\end{array}$ & $\begin{array}{l}1.0514082919738793229 \\
.01928202831056145067\end{array}$ \\
\hline .001 & - & - & $\begin{array}{r}1.232466849 \\
.006400460\end{array}$ & $\begin{array}{r}1.051794454929 \\
.001936923346\end{array}$ \\
\hline 0.000 & - & - & $\begin{array}{l}1.2337 \\
0 .\end{array}$ & $\begin{array}{l}1.051799789 \\
0 .\end{array}$ \\
\hline
\end{tabular}




\section{Summation of $T_{p}$ AND $U_{p}, p=2$ AND $p=3$}

We first take up the series $\left(1.8_{p}\right)$. We expand the ratio of hyperbolic cosines as follows:

$$
\frac{\cosh (2 k+1) x}{\cosh (2 k+1) b}=\sum_{n=0}^{\infty}(-1)^{n}\left\{e^{-(2 k+1)[(2 n+1) b-x]}+e^{-(2 k+1)[(2 n+1) b+x]}\right\} .
$$

Then, upon using again the Laplace transform technique (2.1), (2.2), and interchanging the summations over $k$ and $n$, one obtains after an elementary calculation

$$
T_{p}(x, b)=\frac{1}{2^{p}(p-1) !} \sum_{n=0}^{\infty}(-1)^{n} e^{(2 n+1) b}\left[\varphi_{n}(-x)+\varphi_{n}(x)\right],
$$

where

$$
\varphi_{n}(s)=e^{s} \int_{0}^{1} \frac{w_{p}(t) d t}{e^{2[(2 n+1) b+s]}-t}, \quad-b \leq s \leq b .
$$

The integral in (5.2) again is a Stieltjes transform of the weight function (2.8), this time evaluated at $u=\exp (2[(2 n+1) b+s])$. Clearly, $u>1$, unless $n=0$ and $s=-b$, in which case, by (2.7) and (1.7),

$$
\varphi_{0}(-b)=e^{-b} \int_{0}^{1} \frac{w_{p}(t)}{1-t} d t=\left(2^{p}-1\right)(p-1) ! \zeta(p) e^{-b} .
$$

The integral in (5.2), hence both $\varphi_{n}(x)$ and $\varphi_{n}(-x)$ in (5.1) (the latter if $n>0$ or $x<b)$, can be computed, as before, by the recursive procedure (2.12), (2.13) (where $z^{-2}$ is to be replaced by $u$ ). For large $n$, this procedure converges almost instantaneously.

The series in (5.1), on the other hand, converges geometrically, with ratio $\exp (-2 b)$. This is easily seen by noting that its general term (including the factor in front of the series) behaves like $2(-1)^{n} \cosh x \cdot e^{-2 n b}$ as $n \rightarrow \infty$. Thus, convergence is quite satisfactory, unless $b$ is small, the speed of convergence being independent of $x$. Table 5.1 shows the number of terms, $N$, required

TABLE 5.1

Number of terms required in the series of (5.1) to achieve an accuracy of acc significant decimal digits

\begin{tabular}{|crrrrrrrrrrr|}
\hline$b$ & $a c c$ & $p=2$ & $p=3$ & $b$ & $a c c$ & $p=2$ & $p=3$ & $b$ & $a c c$ & $p=2$ & $p=3$ \\
\hline .05 & 4 & 104 & 105 & .20 & 4 & 26 & 26 & .80 & 4 & 7 & 7 \\
& 8 & 196 & 198 & & 8 & 49 & 49 & & 8 & 13 & 13 \\
& 12 & 288 & 290 & & 12 & 72 & 72 & & 12 & 18 & 18 \\
& 16 & 380 & 382 & & 16 & 95 & 96 & & 16 & 24 & 24 \\
& 20 & 473 & 474 & & 20 & 118 & 119 & & 20 & 30 & 30 \\
.10 & 4 & 52 & 53 & .40 & 4 & 13 & 13 & 1.60 & 4 & 4 & 4 \\
& 8 & 98 & 99 & & 8 & 25 & 25 & & 8 & 6 & 6 \\
& 12 & 144 & 145 & & 12 & 36 & 36 & & 12 & 9 & 9 \\
& 16 & 190 & 191 & & 16 & 48 & 48 & & 16 & 12 & 12 \\
& 20 & 236 & 237 & & 20 & 59 & 59 & & 20 & 15 & 15 \\
\hline
\end{tabular}


in (5.1) to achieve various accuracies. As mentioned, $N$ does not depend on $x$. It can be seen that the convergence characteristics of the series are virtually the same for $p=2$ and $p=3$. (When $x$ is very close to $b$, the backward recursion (2.12) with $\nu \leq 99$ for evaluating $\varphi_{0}(-x)$ in (5.1) may provide only limited accuracy; cf. Example 1.)

For the series $\left(1.9_{p}\right)$ one finds similarly

$$
U_{p}(x, b)=\frac{1}{2^{p}(p-1) !} \sum_{n=0}^{\infty}(-1)^{n} e^{(2 n+1) b}\left[\varphi_{n}(-x)-\varphi_{n}(x)\right],
$$

with $\varphi_{n}(\cdot)$ defined in $(5.2)$; the convergence behavior, when $x>0$, is similar to the one shown in Table 5.1 for the series (5.1).

Series of the types $\left(1.8_{p}\right),\left(1.9_{p}\right)$, which include alternating sign factors, can be treated similarly.

\section{APPENDIX}

Recursion coefficients $\alpha_{n}, \beta_{n}$ for the (monic) polynomials $\left\{\pi_{k}\left(\cdot ; w_{2}\right)\right\}$ and $\left\{\pi_{k}\left(\cdot ; w_{3}\right)\right\}$ orthogonal on $[0,1]$ with respect to the weight functions $w_{2}(t)=$ $t^{-1 / 2} \ln (1 / t)$ and $w_{3}(t)=t^{-1 / 2}[\ln (1 / t)]^{2}$.

\section{TABLE 1}

Recursion coefficients for the polynomials $\left\{\pi_{k}\left(\cdot ; w_{2}\right)\right\}$

$\mathrm{n}$ alpha (n)

$0.1111111111111111111111111 \mathrm{D}+00$ $0.4661483641075477810171688 D+00$ $0.4880690581976426561739654 \mathrm{D}+00$ $0.4938743419208057331274822 D+00$ $0.4962639578613459263700277 \mathrm{D}+00$ $0.4974805136345470499404327 D+00$ $0.4981846424539394712819088 \mathrm{D}+00$ $0.4986290336259843770529448 \mathrm{D}+00$ $0.4989276082849235415546195 \mathrm{D}+00$ $0.4991379564664850047980802 D+00$ $0.4992917697449965925976574 \mathrm{D}+00$ $0.4994076708859089483520375 \mathrm{D}+00$ $0.4994971916094638566242202 D+00$ $0.4995677851751364266156599 \mathrm{D}+00$ $0.4996244439462620957645566 \mathrm{D}+00$ $0.4996706145979840808548842 \mathrm{D}+00$ $0.4997087392464561491728915 \mathrm{D}+00$ $0.4997405876531736626190872 \mathrm{D}+00$ $0.4997674679010946606417245 \mathrm{D}+00$ $0.4997903638440694047466612 \mathrm{D}+00$ $0.4998100270509691381359106 \mathrm{D}+00$ $0.4998270396901901811053116 \mathrm{D}+00$ $0.4998418584011269547061628 \mathrm{D}+00$ $0.4998548454525784424706020 \mathrm{D}+00$ $0.4998662912324218943301592 \mathrm{D}+00$ $0.4998764307204424397405948 D+00$ $0.4998854557169246669449019 D+00$ $0.4998935240328226886591572 \mathrm{D}+00$ $0.4999007664750365107918077 D+00$ $0.4999072922115382430628671 \mathrm{D}+00$ $0.4999131929321822564939469 \mathrm{D}+00$ $0.4999185461046623069180692 \mathrm{D}+00$ $0.4999234175438090965764987 \mathrm{D}+00$ $0.4999278634549460876866086 \mathrm{D}+00$ $0.4999319320708932838633869 \mathrm{D}+00$ $\operatorname{beta}(n)$

$0.4000000000000000000000000 D+01$ $0.2765432098765432098765432 \mathrm{D}-01$ $0.5534292684170711183265476 \mathrm{D}-01$ $0.5940526298488865183067045 \mathrm{D}-01$ $0.6077714606674732893827287 \mathrm{D}-01$ $0.6140371143126410746951299 D-01$ $0.6174167659202270796881379 D-01$ $0.6194453627914717711328688 \mathrm{D}-01$ $0.6207576580933626144340940 \mathrm{D}-01$ $0.6216550244588411861001340 \mathrm{D}-01$ $0.6222955193630939853437094 \mathrm{D}-01$ $0.6227685326078544899112281 D-01$ $0.6231277082877488477563886 \mathrm{D}-01$ $0.6234068199929453251339864 \mathrm{D}-01$ $0.6236279899520571298076283 \mathrm{D}-01$ $0.6238061988995864213325718 \mathrm{D}-01$ $0.6239518834725249753338138 D-01$ $0.6240724948356001710689111 D-01$ $0.6241734675384434899468191 \mathrm{D}-01$ $0.6242588405027882485913615 \mathrm{D}-01$ $0.6243316658846858305615025 D-01$ $0.6243942847487754986053115 D-01$ $0.6244485169209210663968073 D-01$ $0.6244957942452422353437694 \mathrm{D}-01$ $0.6245372557342242600457226 \mathrm{D}-01$ $0.6245738165737186124658778 \mathrm{D}-01$ $0.6246062188808478979166957 D-01$ $0.6246350695269707453479568 D-01$ $0.6246608686595204798542240 D-01$ $0.6246840314472866468672816 \mathrm{D}-01$ $0.6247049048282836967879567 \mathrm{D}-01$ $0.6247237805306714282029063 \mathrm{D}-01$ $0.6247409052851061161429977 D-01$ $0.6247564888999735836355421 \mathrm{D}-01$ $0.62477071059564418235950530-01$ 


\section{TABLE 1 (continued)}

alpha (n)

$0.4999356649724540623274387 \mathrm{D}+00$ $0.4999390981604717422591136 \mathrm{D}+00$ $0.4999422629314923704386477 \mathrm{D}+00$ $0.4999451865971175754297572 \mathrm{D}+00$ $0.4999478930781540114073128 \mathrm{D}+00$ $0.4999504033978688392463883 \mathrm{D}+00$ $0.4999527360934751182615016 \mathrm{D}+00$ $0.4999549075609863005157917 \mathrm{D}+00$ $0.4999569323454961738619833 \mathrm{D}+00$ $0.4999588233865400022085640 \mathrm{D}+00$ $0.4999605922263117426170318 \mathrm{D}+00$ $0.4999622491870299058307070 \mathrm{D}+00$ $0.4999638035225698808616725 \mathrm{D}+00$ $0.4999652635485445800661969 D+00$ $0.4999666367542657434951760 \mathrm{D}+00$ $0.4999679298994151058697458 \mathrm{D}+00$ $0.4999691490977670252895535 \mathrm{D}+00$ $0.4999702998899082096191761 D+00$ $0.4999713873065772549159492 \mathrm{D}+00$ $0.4999724159239822749292821 \mathrm{D}+00$ C. $4999733899122375046656649 \mathrm{D}+00$ $0.4999743130778803590509507 \mathrm{D}+00$ $0.4999751889012818397363903 \mathrm{D}+00$ $0.4999760205696396844641164 \mathrm{D}+00$ $0.4999768110061406606236641 \mathrm{D}+00$ $0.4999775628957922328764517 \mathrm{D}+00$ $0.4999782787083515100262005 \mathrm{D}+00$ $0.4999789607187184893133689 \mathrm{D}+00$ $0.4999796110251092079375052 \mathrm{D}+00$ $0.4999802315652808832931335 \mathrm{D}+00$ $0.4999808241310441662847611 \mathrm{D}+00$ $0.4999813903812661709776500 \mathrm{D}+00$ $0.4999819318535410926892670 \mathrm{D}+00$ $0.4999824499746822529848129 \mathrm{D}+00$ $0.4999829460701697066273291 D+00$ $0.4999834213726706073831202 \mathrm{D}+00$ $0.4999838770297349356284462 \mathrm{D}+00$ $0.4999843141107565888762890 \mathrm{D}+00$ $0.4999847336132789314898947 \mathrm{D}+00$ $0.4999851364687144438985805 \mathrm{D}+00$ $0.4999855235475398955558700 \mathrm{D}+00$ $0.4999858956640213131307627 \mathrm{D}+00$ $0.4999862535805167765288282 \mathrm{D}+00$ $0.4999865980113996234652464 \mathrm{D}+00$ $0.4999869296266398705890535 \mathrm{D}+00$ $0.4999872490550774736510815 \mathrm{D}+00$ $0.4999875568874173723953749 \mathrm{D}+00$ $0.4999878536789730305318126 \mathrm{D}+00$ $0.4999881399521823296847290 \mathrm{D}+00$ $0.4999884161989171590890288 \mathrm{D}+00$ $0.4999886828826058173914092 \mathrm{D}+00$ $0.4999889404401853724423155 \mathrm{D}+00$ $0.4999891892838993776656731 D+00$ $0.4999894298029547919706246 \mathrm{D}+00$ $0.4999896623650505703804859 \mathrm{D}+00$ $0.4999898873177891633918131 \mathrm{D}+00$ $0.4999901049899810715268292 \mathrm{D}+00$ $0.4999903156928516094091928 \mathrm{D}+00$ $0.4999905197211581872778772 \mathrm{D}+00$ $0.4999907173542256001838438 \mathrm{D}+00$ $0.4999909088569061417086197 \mathrm{D}+00$ $0.4999910944804707157151021 \mathrm{D}+00$ $0.4999912744634365583230273 \mathrm{D}+00$ $0.4999914490323366734035746 \mathrm{D}+00$ $0.4999916184024356271670790 \mathrm{D}+00$ $\operatorname{beta}(n)$

$0.6247837246679940094973984 \mathrm{D}-01$ $0.6247956636600570708844248 \mathrm{D}-01$ $0.6248066427536794284683246 \mathrm{D}-01$ $0.6248167620434672378022245 \mathrm{D}-01$ $0.6248261089183034006005443 D-01$ $0.6248347599478382249321288 \mathrm{D}-01$ $0.6248427824502116118751316 \mathrm{D}-01$ $0.6248502358010969536816872 \mathrm{D}-01$ $0.6248571725317097460386049 \mathrm{D}-01$ $0.6248636392537765834044482 \mathrm{D}-01$ $0.6248696774419352905015697 \mathrm{D}-01$ $0.6248753240981319504469108 \mathrm{D}-01$ $0.6248806123179200596514722 \mathrm{D}-01$ $0.6248855717748684742433619 D-01$ $0.6248902291363342497098043 D-01$ $0.6248946084214908332126385 \mathrm{D}-01$ $0.6248987313105962943007473 \mathrm{D}-01$ $0.6249026174129439284484899 \mathrm{D}-01$ $0.6249062844996838433907696 \mathrm{D}-01$ $0.6249097487066807275398102 \mathrm{D}-01$ $0.6249130247117342087934172 \mathrm{D}-01$ $0.6249161258897980601024296 \mathrm{D}-01$ $0.6249190644492645302489131 D-01$ $0.6249218515519076536316924 \mathrm{D}-01$ $0.6249244974186864670497904 \mathrm{D}-01$ $0.6249270114232811644774820 \mathrm{D}-01$ $0.6249294021749607086844032 D-01$ $0.6249316775921498850954361 \mathrm{D}-01$ $0.6249338449678696015999789 \mathrm{D}-01$ $0.6249359110280602015518800 \mathrm{D}-01$ $0.6249378819836585975999631 D-01$ $0.6249397635771819984114800 \mathrm{D}-01$ $0.6249415611244704749850512 \mathrm{D}-01$ $0.6249432795521547829357330 \mathrm{D}-01$ $0.6249449234313423932856533 D-01$ $0.6249464970079516550903631 D-01$ $0.6249480042300698114169578 D-01$ $0.6249494487726638748190090 \mathrm{D}-01$ $0.6249508340599330177430746 \mathrm{D}-01$ $0.6249521632855562063538542 \mathrm{D}-01$ $0.6249534394310585118905247 \mathrm{D}-01$ $0.6249546652824932049195604 \mathrm{D}-01$ $0.6249558434456138111659900 \mathrm{D}-01$ $0.6249569763596903056671454 \mathrm{D}-01$ $0.6249580663101061396102570 \mathrm{D}-01$ $0.6249591154398574866660389 \mathrm{D}-01$ $0.6249601257600626690066200 \mathrm{D}-01$ $0.6249610991595779263883089 \mathrm{D}-01$ $0.6249620374138053097068322 \mathrm{D}-01$ $0.6249629421927693288786306 \mathrm{D}-01$ $0.6249638150685309052629656 \mathrm{D}-01$ $0.6249646575220000346266933 \mathrm{D}-01$ $0.6249654709492022401174982 \mathrm{D}-01$ $0.6249662566670482840875654 \mathrm{D}-01$ $0.6249670159186516247938764 \mathrm{D}-01$ $0.6249677498782336725853075 \mathrm{D}-01$ $0.6249684596556529537947903 D-01$ $0.6249691463005907714323314 \mathrm{D}-01$ $0.6249698108064228095881835 D-01$ $0.6249704541138033192705538 D-01$ $0.6249710771139860088117153 \mathrm{D}-01$ $0.6249716806519035083547536 \mathrm{D}-01$ $0.6249722655290252557984423 \mathrm{D}-01$ $0.6249728325060118350439253 \mathrm{D}-01$ $0.624973382 ; 51821636937157 \mathrm{D}-01$ 
TABLE 2

Recursion coefficients for the polynomials $\left\{\pi_{k}\left(\cdot ; w_{3}\right)\right\}$

n alpha (n)

$0.37037037037037037037 D-01$ $0.35811288669783060917 \mathrm{D}+00$ $0.44293596764346020311 D+00$ $0.46925333322630284096 \mathrm{D}+00$ $0.48077976287000283670 \mathrm{D}+00$ $0.48684740638240343111 \mathrm{D}+00$ $0.49043291987640869651 D+00$ $0.49272768237210454609 D+00$ $0.49428489903347684509 D+00$ $0.49539008804668943172 \mathrm{D}+00$ $0.49620279412350964003 \mathrm{D}+00$ $0.49681787613674196493 \mathrm{D}+00$ $0.49729461823511554334 \mathrm{D}+00$ $0.49767162298766581034 D+00$ $0.49797490489096250254 \mathrm{D}+00$ $0.49822251490262493942 D+00$ $0.49842729720309510051 D+00$ $0.49859859328823678290 D+00$ $0.49874332901963438146 \mathrm{D}+00$ $0.49886672742402863250 D+00$ $0.49897278759666604684 \mathrm{D}+00$ $0.49906461349768728317 D+00$ $0.49914464410949686541 \mathrm{D}+00$ $0.49921481738564071689 \mathrm{D}+00$ $0.49927668889965310293 D+00$ $0.49933151895604900098 D+00$ $0.49938033739401414223 D+00$ $0.49942399238220700438 D+00$ $0.49946318757058857679 D+00$ $0.49949851066980882148 D+00$ $0.49953045564672500675 \mathrm{D}+00$ $0.49955944011544773239 D+00$ $0.49958581907688664129 D+00$ $0.49960989585754508391 D+00$ $0.49963193088162418308 D+00$ $0.49965214875344531167 \mathrm{D}+00$ $0.49967074401221995461 \mathrm{D}+00$ $0.49968788583618920278 D+00$ $0.49970372190980540385 \mathrm{D}+00$ $0.49971838161991717404 \mathrm{D}+00$ $0.49973197871081541506 \mathrm{D}+00$ $0.49974461350037973769 D+00$ $0.49975637473833709910 D+00$ $0.49976734117120030262 D+00$ $0.49977758286563735392 \mathrm{D}+00$ $0.49978716233197190813 \mathrm{D}+00$ $0.49979613548158744430 D+00$ $0.49980455244572020271 D+00$ $0.49981245827811267119 D+00$ $0.49981989355998203428 D+00$ $0.49982689492252312177 \mathrm{D}+00$ $0.49983349549954855405 \mathrm{D}+00$ $0.49983972532074252462 \mathrm{D}+00$ $0.49984561165426967645 D+00$ $0.49985117930605904348 D+00$ $0.49985645088191383563 D+00$ $0.49986144701763254412 \mathrm{D}+00$ $0.49986618658152699341 \mathrm{D}+00$ $0.49987068685305789539 D+00$ $0.49987496368075360253 D+00$ $0.49987903162211334570 \mathrm{D}+00$ $0.49988290406780632029 D+00$ $0.49988659335214961618 D+00$ $0.49989011085157064972 \mathrm{D}+00$ $0.49989346707252485814 \mathrm{D}+00$ $\operatorname{beta}(n)$

$0.16000000000000000000 D+02$ $0.66282578875171467764 \mathrm{D}-02$ $0.41154551017361395415 \mathrm{D}-01$ $0.51741782003936091009 \mathrm{D}-01$ $0.56064845754865753829 D-01$ $0.58228578157740470460 \mathrm{D}-01$ $0.59461384764548385798 D-01$ $0.60229080854911299451 D-01$ $0.60739066803493998597 \mathrm{D}-01$ $0.61094906789182697675 \mathrm{D}-01$ $0.61352955641236678499 D-01$ $0.61545998375888748886 D-01$ $0.61694155907229941430 \mathrm{D}-01$ $0.61810329736885846614 \mathrm{D}-01$ $0.61903100105428254212 \mathrm{D}-01$ $0.61978352734467430133 D-01$ $0.62040233674523126765 \mathrm{D}-01$ $0.62091731596138376834 \mathrm{D}-01$ $0.62135044810738308068 \mathrm{D}-01$ $0.62171819377072260690 \mathrm{D}-01$ $0.62203307551568758377 \mathrm{D}-01$ $0.62230475638549453317 \mathrm{D}-01$ $0.62254078896209909882 \mathrm{D}-01$ $0.62274714515063386935 \mathrm{D}-01$ $0.62292859708354606101 D-01$ $0.62308899510226347107 D-01$ $0.62323147341070148965 \mathrm{D}-01$ $0.62335860413249654858 \mathrm{D}-01$ $0.62347251405111280344 \mathrm{D}-01$ $0.62357497401578225918 \mathrm{D}-01$ $0.62366746808956619876 \mathrm{D}-01$ $0.62375124751988228320 \mathrm{D}-01$ $0.62382737322232212745 \mathrm{D}-01$ $0.62389674948882016821 \mathrm{D}-01$ $0.62396015093213554691 D-01$ $0.62401824417420930673 D-01$ $0.62407160541823668615 \mathrm{D}-01$ $0.62412073477358958151 D-01$ $0.62416606800160811616 \mathrm{D}-01$ $0.62420798619957045131 D-01$ $0.62424682382629097021 \mathrm{D}-01$ $0.62428287538611475284 \mathrm{D}-01$ $0.62431640102160245772 \mathrm{D}-01$ $0.62434763121387056841 D-01$ $0.62437677074965693541 D-01$ $0.62440400208298018187 \mathrm{D}-01$ $0.62442948819471456645 \mathrm{D}-01$ $0.62445337503398093115 \mathrm{D}-01$ $0.62447579360980611775 D-01$ $0.62449686178915178922 \mathrm{D}-01$ $0.62451668584748955026 \mathrm{D}-01$ $0.62453536181008802223 \mathrm{D}-01$ $0.62455297661568124452 \mathrm{D}-01$ $0.62456960912889718816 \mathrm{D}-01$ $0.62458533102349863062 \mathrm{D}-01$ $0.62460020755493639085 \mathrm{D}-01$ $0.62461429823778722643 \mathrm{D}-01$ $0.62462765744122685831 \mathrm{D}-01$ $0.62464033491367810709 D-01$ $0.62465237624609943064 \mathrm{D}-01$ $0.62466382328197957392 \mathrm{D}-01$ $0.62467471448093066886 \mathrm{D}-01$ $0.62468508524178538288 \mathrm{D}-01$ $0.62469496819027145677 \mathrm{D}-01$ $0.62470439343563310277 \mathrm{D}-01$ 
TABLE 2 (continued)

$\begin{array}{ccc}\mathrm{n} & \text { alpha }(\mathrm{n}) & \text { beta }(\mathrm{n}) \\ 65 & 0.49989667173013992394 \mathrm{D}+00 & 0.62471338879997137574 \mathrm{D}-01 \\ 66 & 0.49989973381868792364 \mathrm{D}+00 & 0.62472198002356840682 \mathrm{D}-01 \\ 67 & 0.49990266167484177651 \mathrm{D}+00 & 0.62473019094902672552 \mathrm{D}-01 \\ 68 & 0.49990546303454826112 \mathrm{D}+00 & 0.62473804368668536520 \mathrm{D}-01 \\ 69 & 0.49990814508424340115 \mathrm{D}+00 & 0.62474555876345704732 \mathrm{D}-01 \\ 70 & 0.49991071450704447675 \mathrm{D}+00 & 0.62475275525695845215 \mathrm{D}-01 \\ 71 & 0.49991317752447402614 \mathrm{D}+00 & 0.62475965091657110617 \mathrm{D}-01 \\ 72 & 0.49991553993420306482 \mathrm{D}+00 & 0.62476626227286812920 \mathrm{D}-01 \\ 73 & 0.49991780714424177721 \mathrm{D}+00 & 0.62477260473666718445 \mathrm{D}-01 \\ 74 & 0.49991998420395478897 \mathrm{D}+00 & 0.62477869268881843823 \mathrm{D}-01 \\ 75 & 0.49992207583223368018 \mathrm{D}+00 & 0.62478453956170477586 \mathrm{D}-01 \\ 76 & 0.49992408644312069750 \mathrm{D}+00 & 0.62479015791331707690 \mathrm{D}-01 \\ 77 & 0.49992602016914386125 \mathrm{D}+00 & 0.62479555949466760887 \mathrm{D}-01 \\ 78 & 0.49992788088259415486 \mathrm{D}+00 & 0.62480075531121750293 \mathrm{D}-01 \\ 79 & 0.49992967221494964853 \mathrm{D}+00 & 0.62480575567891808625 \mathrm{D}-01 \\ 80 & 0.49993139757462874885 \mathrm{D}+00 & 0.62481057027539907959 \mathrm{D}-01 \\ 81 & 0.49993306016323485719 \mathrm{D}+00 & 0.62481520818677805912 \mathrm{D}-01 \\ 82 & 0.49993466299043719936 \mathrm{D}+00 & 0.62481967795051404622 \mathrm{D}-01 \\ 83 & 0.49993620888761714558 \mathrm{D}+00 & 0.62482398759468269883 \mathrm{D}-01 \\ 84 & 0.49993770052039570566 \mathrm{D}+00 & 0.62482814467401053793 \mathrm{D}-01 \\ 85 & 0.49993914040014582863 \mathrm{D}+00 & 0.62483215630297026601 \mathrm{D}-01 \\ 86 & 0.49994053089458246073 \mathrm{D}+00 & 0.62483602918620793101 \mathrm{D}-01 \\ 87 & 0.49994187423751384843 \mathrm{D}+00 & 0.62483976964654494941 \mathrm{D}-01 \\ 88 & 0.49994317253782916515 \mathrm{D}+00 & 0.62484338365077338409 \mathrm{D}-01 \\ 89 & 0.49994442778779006308 \mathrm{D}+00 & 0.62484687683344099460 \mathrm{D}-01 \\ 90 & 0.49994564187068709249 \mathrm{D}+00 & 0.62485025451880310790 \mathrm{D}-01 \\ 91 & 0.49994681656791599301 \mathrm{D}+00 & 0.62485352174110100732 \mathrm{D}-01 \\ 92 & 0.49994795356552355925 \mathrm{D}+00 & 0.62485668326331105650 \mathrm{D}-01 \\ 93 & 0.49994905446026804320 \mathrm{D}+00 & 0.62485974359449494323 \mathrm{D}-01 \\ 94 & 0.49995012076523481262 \mathrm{D}+00 & 0.62486270700586905479 \mathrm{D}-01 \\ 95 & 0.49995115391504418198 \mathrm{D}+00 & 0.62486557754569991412 \mathrm{D}-01 \\ 96 & 0.49995215527068492031 \mathrm{D}+00 & 0.62486835905312266817 \mathrm{D}-01 \\ 97 & 0.49995312612400387501 \mathrm{D}+00 & 0.62487105517097069732 \mathrm{D}-01 \\ 98 & 0.49995406770187939389 \mathrm{D}+00 & 0.62487366935769639540 \mathrm{D}-01 \\ 99 & 0.49995498117010374556 \mathrm{D}+00 & 0.62487620489845595326 \mathrm{D}-01\end{array}$

\section{BIBLIOGRAPHY}

1. K. M. Dempsey, D. Liu, and J. P. Dempsey, Plana's summation formula for $\sum_{m=1,3, \ldots}^{\infty}$ $m^{-2} \sin (m \alpha), m^{-3} \cos (m \alpha), m^{-2} A^{m}, m^{-3} A^{m}$, Math. Comp. 55 (1990), 693-703.

2. W. Gautschi, On the preceding paper "A Legendre polynomial integral" by James $L$. Blue, Math. Comp. 33 (1979), 742-743.

3. _ Minimal solutions of three-term recurrence relations and orthogonal polynomials, Math. Comp. 36 (1981), 547-554.

4. __, On generating orthogonal polynomials, SIAM J. Sci. Statist. Comput. 3 (1982), 289317.

5. __ Questions of numerical condition related to polynomials, Studies in Numerical Analysis (G. H. Golub, ed.), Math. Assoc. Amer., 1984, pp. 140-177.

6. _ A class of slowly convergent series and their summation by Gaussian quadrature, Math. Comp. 57 (1991), 309-324.

7. W. Gautschi and G. V. Milovanović, Gaussian quadrature involving Einstein and Fermi functions with an application to summation of series, Math. Comp. 44 (1985), 177-190.

8. E. R. Hansen, A table of series and products, Prentice-Hall, Englewood Cliffs, NJ, 1975.

9. A. McLellan IV, Tables of the Riemann zeta function and related functions, Math. Comp. 22 (1968), Review 69, 687-688.

Department of Computer Sciences, Purdue University, West Lafayette, Indiana 47907 E-mail address:wxg@cs.purdue.edu 